\title{
DataCloud Infrastructure to manage FAIR Environmental Data
}

\author{
F. Aguilar, ${ }^{a}$ \\ ${ }^{a}$ IFCA-CSIC, \\ Av. de los Castros s/n. Ed Juan Jorda. Santander (Cantabria), Spain \\ E-mail: aguilarf@ifca.unican.es
}

\begin{abstract}
The use of Jupyter notebooks as a platform for data management is becoming popular in different disciplines thanks to the features provided: web-based interface, code running capabilities, customization, kernel configuration, etc. However, it is sometimes limited to the server capacity, so working with big datasets is difficult since certain data analysis requires intensive computing or even GPUs. Thanks to diverse Cloud Computing-based solutions, specially those provided by eXtreme-DataCloud project, these limitations can be solved, providing an integrated environment where storage and computing resources could support a system in a transparent way for the user.
\end{abstract}

KeYwords: Computing, Data processing methods, Software architectures

${ }^{1}$ Corresponding author. 


\section{Contents}

1 Introduction 1

$\begin{array}{lll}1.1 & \text { Motivation } & 2\end{array}$

1.1.1 Use Case $\quad 3$

2 System Components 3

2.0.1 Jupyter HUB $\quad 3$

2.0.2 Onedata 4

2.0.3 INDIGO PaaS Orchestrator $\quad 5$

$\begin{array}{lll}2.0 .4 & \text { AAI as integrator } & 6\end{array}$

3 Architecture 6

$\begin{array}{ll}3.1 \text { Configuration } & 7\end{array}$

$\begin{array}{llr}4 & \text { Conclusions } & 8\end{array}$

$\begin{array}{ll}\text { References } & 9\end{array}$

\section{Introduction}

In many scientific disciplines, there has always been the need for smart environments where different software components were accessible as well as data management functionalities (access, analysis, visualization). However, during the last years, the access to "Big Data" sources has been extended and desktop resources are not yet valid to address the problems derived from data in terms of volume, variety, velocity or veracity. For this reason, new scientific gateways are being used to access to complex computing resources in a cloud-based fashion, such as Jupyter Notebooks [1], which support different types of kernels in diverse programming languages for different purposes. The catalogue of available Jupyter Notebooks is increasing and there are several examples in many scientific domains [2] [3].

Jupyter supports different deployment methods, and the Jupyter Hub version set up a flexible, customizable and scalable environment to manage different users. Thanks to technologies like Docker [4], users access via Jupyter Hub to his/her own computer environment with specific software and Jupyter notebooks installed. However, the available resources are limited by the hardware, but it can be solved integrating Jupyter with different cloud-based systems, like storage or workload managers.

Authentication and Authorization Infrastructures and standards like OpenID Connect (OIDC) [5], supported by Jupyter, can be used in order to integrate those systems in a transparent way. This work describes how the OIDC standard is used within Jupyter Hub environment to provide, thanks to cloud computing services, a complete system (DataCloud) to access to distributed storage (Onedata 
[6]) as well as workload managers (PaaS Orchestrator [7]) to run jobs with specific computing requirements. This complete solution, adaptable to many purposes, facilitates the data access in a FAIR way [8], not only via Jupyter notebooks but also in computing worker nodes and desktops. Besides, it enables data processing with specific hardware requirements via job submission, being the output directly accessible from the notebook.

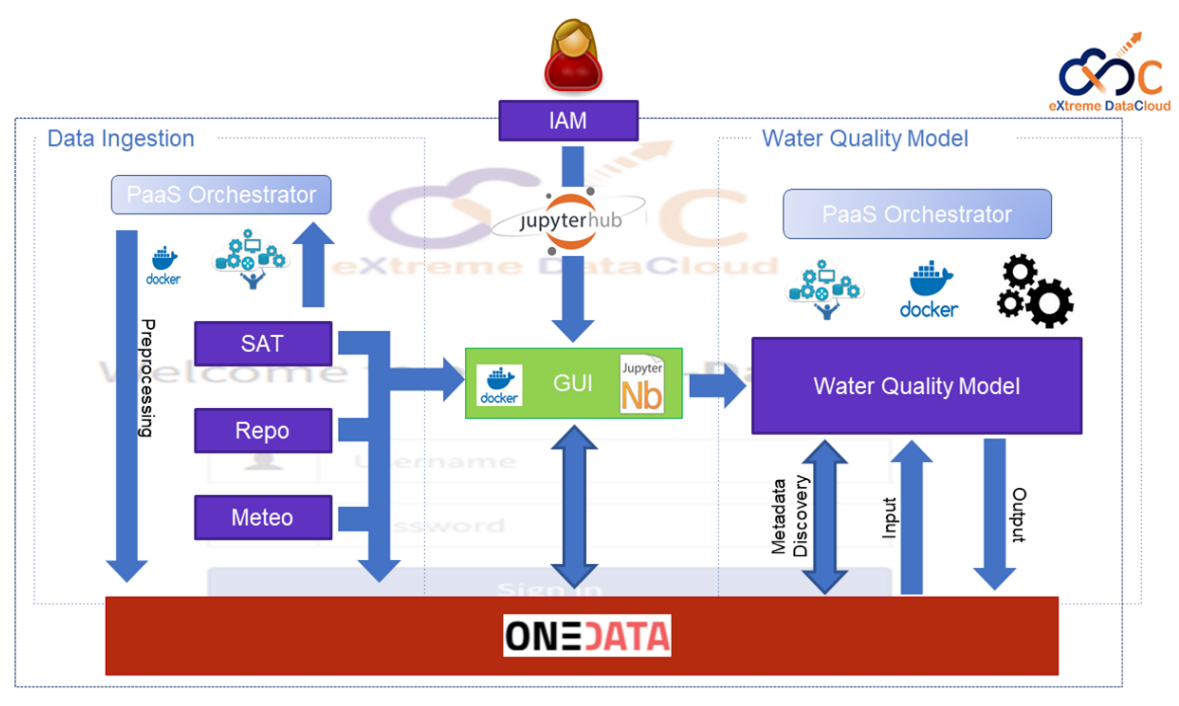

Figure 1. DataCloud components in the architecture

\subsection{Motivation}

Diverse initiatives at European [9] at the international level are promoting the use of open data for reuse in research. In order to make the data useful, it must be published following the FAIR principles (Findable, Accessible, Interoperable and Reusable), supported by well-described metadata. These recommendations aim to ensure the scientific data quality to be reused in a different context, getting an added value as well as new ways to use the data. However, the data is not always easily available for researchers with limited computing or storage resources.

In parallel, the use of Jupyter Notebooks not only as teaching purposes [10] but also for data management and analysis development is becoming popular in the scientific community. It is not limited to Python itself, but it can support different programming languages like R, perl or even Matlab. However, although Jupyter can serve as a complete-enough application for many disciplines, it is limited to the resources provided by the hardware behind.

Supported by Cloud computing solutions oriented to provide services, the features of the Jupyter notebooks can be improved and completed in order to extend the system oriented to satisfy the needs of users in terms of storage and computing. The proposed solution offers a completeenough environment to get access to a distributed storage space, available not only from the Jupyter server itself but also from different access points (desktop, web interface, etc.). Also, for those tasks requiring intensive computing resources, a job management system is provided with customizable access to data centres. Finally, in order to manage correctly the users, Authentication 
and Authorization Infrastructures are required and it needs to be interoperable across the different tools.

\subsubsection{Use Case}

The eXtreme-DataCloud project (XDC), under the umbrella of the H2020 programme, aims at developing a scalable environment for data management and computing, addressing the problems of the growing data volume and focused in providing a complete framework for research communities through the European Open Science Cloud. The target of this project is to integrate different services and tools based on Cloud Computing to manage Big Data sources, and Use Cases from diverse disciplines are represented. One of the goals of the project is to deal with extremely large and heterogeneous datasets, including diverse data and metadata types, formats and standards that enable the automatic integration of Big Data.

One of the XDC Use Cases representing LifeWatch ERIC, the European Research Infrastructure for Ecosystems and Biodiversity, is integrating data from those heterogeneous data sources for Environmental data such as Satellites (NASA Landsat, ESA Sentinel), meteorological stations (both historical and forecasting data) or In-situ instrumentation. These sources produce data in different formats like NetCDF4, HDF5 or CSV, and they are accessible via different types of APIs. The goal of this Use Case is to automatize different stages of the data lifecycle in order to simulate freshwater environments like reservoirs to forecast the hydrodynamics and water quality, facing the problem of eutrophication [11].

In order to interoperate those big data sources, the adoption of the four FAIR principles is needed. The use of metadata standards like the Ecological Metadata Language is the way proposed to interoperate the different data sources, and the use of Cloud Computing solutions provided by $\mathrm{XDC}$ the way to manage the complex data life cycle in a FAIR manner: ingestion, curation, analysis, modeling, publishing, etc.

\section{System Components}

The proposed system integrates different components:

- Jupyter Hub and notebooks to act as a user interface.

- Onedata to provide a distributed storage space.

- INDIGO PaaS Orchestrator to run analysis jobs.

- Authentication and Authorization Infrastructure (AAI) to manage different users (INDIGO IAM).

All of them are independent software but they can be integrated thanks to the AAI.

\subsubsection{Jupyter HUB}

JupyterHub extends the capacity of the notebooks with new features oriented to user management. It can support a number of user under the same resources, but providing a closed environment 
and workspace for each user. However, the hardware resources behind are shared and the different configuration options allow system administrators to enable one single node or to add any required nodes, making Jupyter a well-scalable tool.

Since the access point is a web interface, Jupyter is available everywhere through a web browser, so no special configuration or software installation is required. The user only need to access to the URL to have access to a pre-configured environment that can be customized if needed, installing new packages or software inside. Furthermore, it is not limited to run Python but it includes dozens of kernels to run different types of programming languages, making Jupyter an adaptable solution.

Finally, there are several possibilities in terms of user management configuration. The users not only can be imported directly from the system but also Jupyter can be connected with external identity providers like social or institutional IDs based on standard protocols like Open ID Connect or SAML. This feature opens Jupyter to entire scientific communities or institutions only adding an specific configuration. The user management will be easier without any required action locally.

Due to these characteristics, Jupyter Hub have been selected for the following reasons:

- Scalable architecture to support scientific communities or institutions.

- Customizable Authentication and Authorization configuration to support already existing Identity Providers.

- Powerful workspaces based on kernels

Also, one of the most interesting features to make Jupyter an interdisciplinar tool is that the workspace assigned to the user is deployed using Docker spawner [12]. Docker supports application packaging including all dependencies in a lightweigth fashion, so a new "Docker Image" can be created including all the components needed to run Jupyter notebooks for any purpose. Whenever a new user access to the system, Jupyter Hub deploys a new instance of that docker Image in order to create a new individual workspace for her/him. The customizable features of Docker enable the integration with different tools, which is exploited to create the proposed system. For example, it can be integrated with a distirbuted storage space like Onedata.

\subsubsection{Onedata}

Onedata is a system oriented to provide a High-performance data management and federation solution offering unified data access across globally distributed environments and multiple types of underlying storage (different technologies), allowing users to share, collaborate and perform computations on the stored data easily as well as transparently. Onedata is distributed in three main components:

- Zones are site federations where storage facilities are post and enable the creation of closed and interconnected communities.

- The Providers are entities that support and expose a concrete volume of available storage.

- The spaces are distributed virtual volumes where user can manage their data. 
From the user point of view, Onedata can be manage with different user interfaces. The spaces are available through a web portal, where users can performed the usual file management actions, like upload, edit, rename, download, delete, etc. The users can also access the data using the command line interface (CLI), which can be installed in a UNIX OS and mount the space as a volume (POSIX compatible). Currently Onedata also exposes different APIs like a CDMI (Cloud Data Management Interface) interface and RESTful interface for integration with other services, which allows developers to directly access and manage spaces, groups, authorization rights and data. It is also compatible with data harvesting protocols like OAI-PMH [13]. From the administrator point of view, as well as the server configuration, Onedata can be managed through the administrator web interface, the OnePanel service. One of the most promising features of Onedata is the metadata management. Onedata comes with extensive support for metadata management, which can be used to describe all kind of resources in Onedata including files, folders, spaces and users.

From the point of view of the integrated system, Onedata is used as a omnipresent storage space that is accessible by the different actors:

- The user themselves can access directly to Onedata to upload or download data using the web interface or any of the Command Line Interfaces.

- Jupyter mounts the Onedata space directly within the Docker container assigned to the user, so the notebook can load data directly.

- The PaaS Orchestrator can also mount Onedata to access to input data and write output data after performing certain action (data analysis, processing).

Due to the scalable characteristics of Onedata, that can be distributed in different computing sites, it is a very good solution to manage data at different volumes. However, it requires a good Internet connection to work properly.

\subsubsection{INDIGO PaaS Orchestrator}

Some exhaustive data analysis or processing requires dedicated and intensive computing resources. For instance, techniques like Deep Learning are being applied to Big Data processing [14] and it needs to be supported by GPUs. In many cases, these resources cannot be dedicated to one single service all the time and they need to be shared under the use of task manager or job-based systems. In this case, extra service is needed to make the use of computing resources closer to Jupyter.

The INDIGO PaaS Orchestrator is a component of the PaaS layer that allows instantiating resources on Cloud Management Frameworks (like OpenStack and OpeNebula) and Mesos clusters. It takes the deployment requests, expressed through templates written in TOSCA YAML Simple Profile [15] defining the specific requirements and characteristics, and deploys them on the best Cloud site available. It is able to deploy a very wide range of applications and services, both temporal and long-standing and any software can be packaged in a Docker container ready to be instantiated in a cloud environment. Thanks to its architecture, INDIGO PaaS Orchestrator can manage the deployment of interconnected processing steps, also known as workflows or pipelines.

The INDIGO PaaS Orchestrator includes an API that allows users to configure the deployment of new jobs in the available environments. Using that API, Jupyter can interact with the PaaS Orchestrator to process data, which output will be stored in Onedata. 


\subsubsection{AAI as integrator}

The final goal of the system is to provide a dedicated workspace per user. Therefore, all the tools and services need to talk to each other in a common language, as well as identify the users to assign dedicated resources. In order to do so, an Authentication and Authorization Infrastructure (AAI) is needed. Whenever a component of the system needs to interchange information with another component, they need to include information about the user they are referring to. In this case, it can be done using tokens. There are different technologies or standards capable to address this functionality. One of the most popular especially for web-based applications is Open ID Connect.

OpenID Connect is an interoperable identity layer implemented on top of the OAuth 2.0 family of specifications. Since it is built on OAuth 2.0, it standardizes the use of this protocol for login and adds a discovery process that enables Identity Provider lookup. OpenID Connect allows clients of all types, including Web-based, mobile, and JavaScript clients, to request and receive information about authenticated sessions and end-users. The specification suite is extensible, allowing participants to use optional features such as encryption of identity data, the discovery of OpenID Providers, and session management. Most of the popular Internet services or portals (GitHub, Google services, etc.) are compatible with these technologies.

Using Open ID connect tokens in all the components of the system is a way to interchange information among them in a secure and complete way.

\section{Architecture}

The components referred in the previous section enables the design of the architecture proposed in Figure 2.

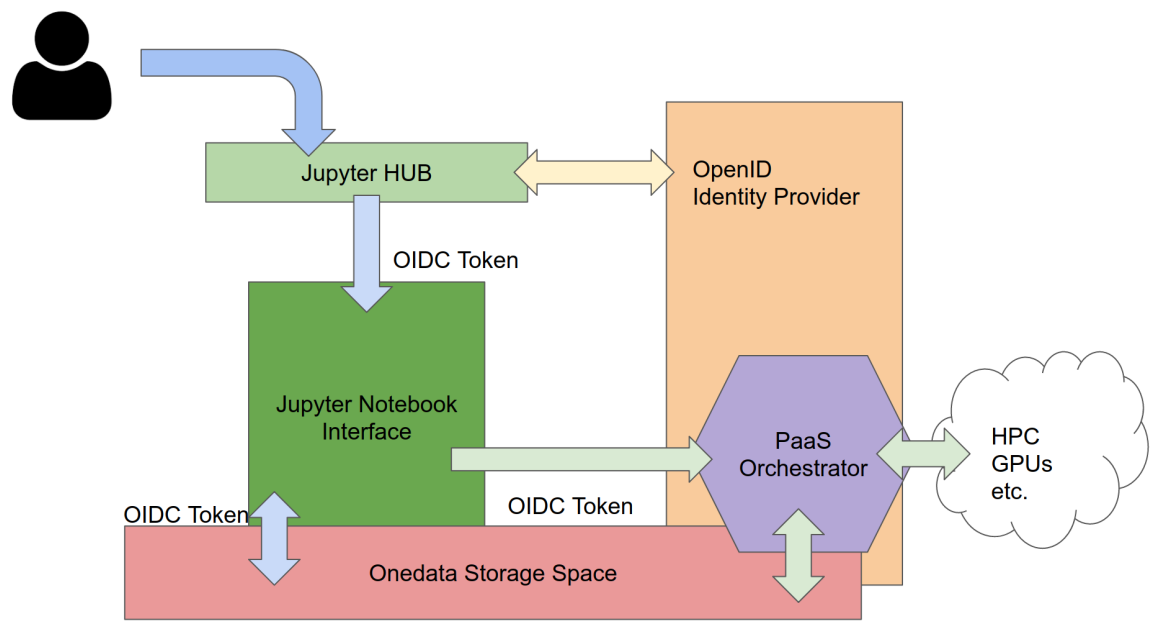

Figure 2. Jupyter + Cloud Computing oriented architecture

The Jupyter Hub is a web-based service, so it is accessed by the user with a web browser. After configuring Jupyter, it is capable to redirect the user to the Open ID connect Identity Provider, that is the place where the user is logged in. Social IDs like Google or Github can be used here, as well 
as institutional identities. Whenever the user is logged, a token is sent to the Jupyter Hub and the information is passed to the Docker deployed as the workspace for the user.

With that token, which can be refreshed asking again to the Identity Provider, the new deployed Docker container can get the credentials needed to mount the user's Onedata space in the same environment. Furthermore, the token can be used to launch a new job in the PaaS Orchestrator, that forward the information to the final computing site and which is also capable to mount Onedata space.

With this architecture, the system works as a whole, providing scalable storage space, access to external computing resources and all the abilities of Jupyter Notebooks and their kernels.

\subsection{Configuration}

In order to set up the described architecture, some specific configuration in Jupyter and its packages need to be done.

First of all, the Jupyter Hub configuration file (default name jupyterhub_config.py) need to be customized to allow Open ID Connect Authentication and Authorization. Listing 1 shows how to do it.

Listing 1. Authenticator configuration

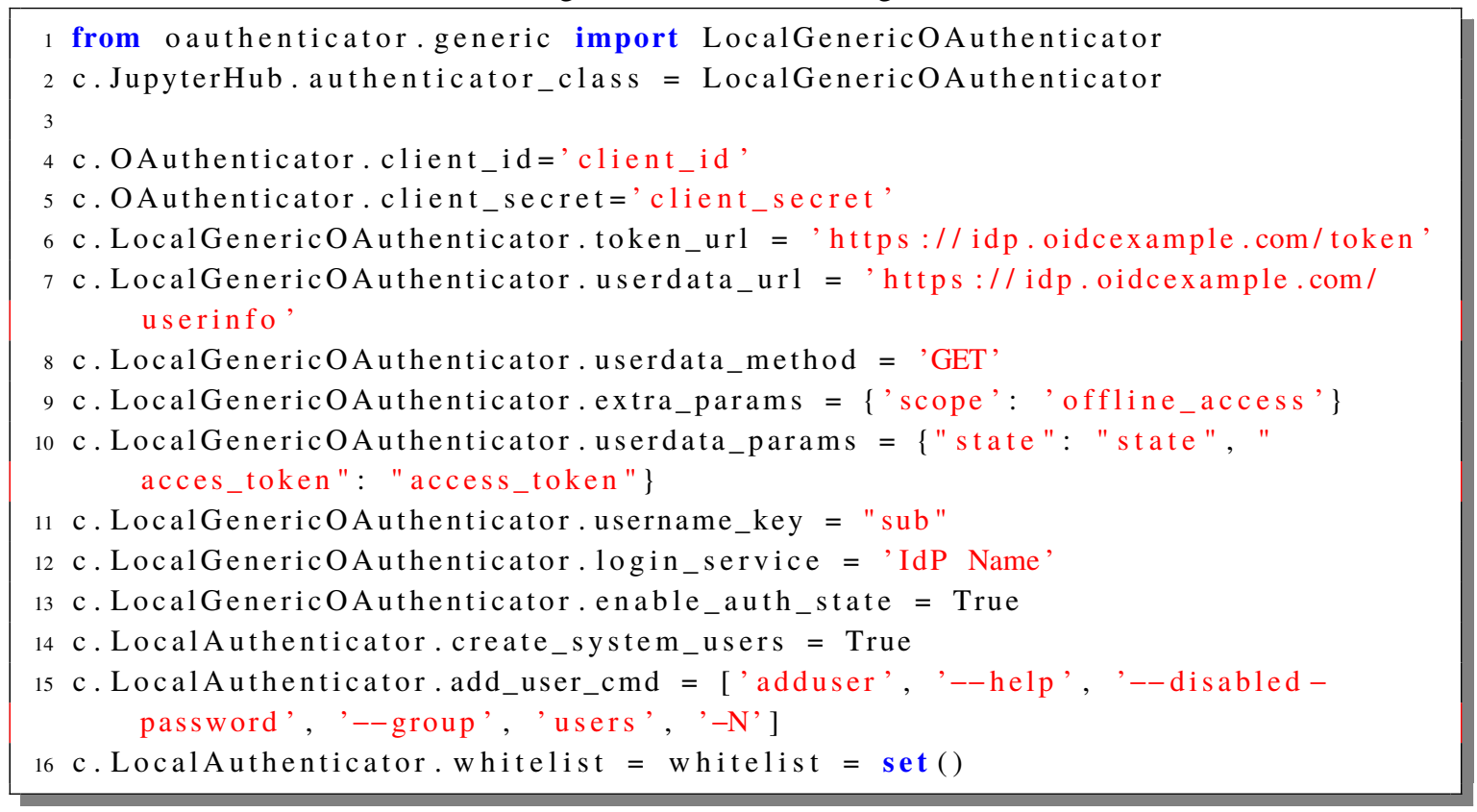

The "OAuthenticator" package need to be imported to support the Open ID standard and the usual configuration items need to be added (client_id, client_secret, token_url, etc.). This means that an Open ID Connect client need to be configured. One particular thing to remark is that we need one of the Open ID parameters to identify the user, that in this case is the "sub" attribute ("c.LocalGenericOAuthenticator.username_key $=$ sub"). Also, we can create or not system users whenever a new user is logged in ("c.LocalAuthenticator.create_system_users = True") and run any command afterwards (“c.LocalAuthenticator.add_user_cmd = ['adduser', '-help', '-disabledpassword', '-group', 'users', '-N']"). Finally, we can define a white list in order to authorize or not the access to the users ("c.LocalAuthenticator. whitelist $=$ whitelist $=\operatorname{set}()$ "). 
The Listing 2 describes the parameters (in the same Jupyter Hub config file) to configure the behaviour of the docker to be launched.

Listing 2. Docker spawner configuration

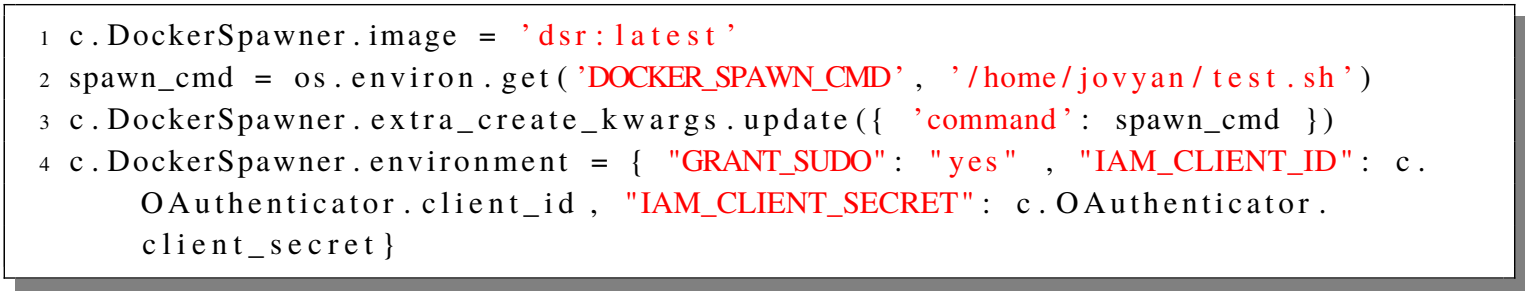

First of all, we need to indicate which Docker image needs to be launched. Notice that this image needs to be built and include all the components needed to run Jupyter notebooks. After that, we can configure which is the command to be run after the docker container is deployed. In this case, it is a customized bash script to mount the Onedata space (using the Open ID token) and to run the Jupyter environment inside. Finally, a set of environmental variables to be included in the docker container deployed can be assigned. They are needed to access Open ID connect parameters within the user workspace.

Finally, a piece of code needs to be updated in order to make everything work. Listing 3 provides more details:

Listing 3. Pre-spawn start function

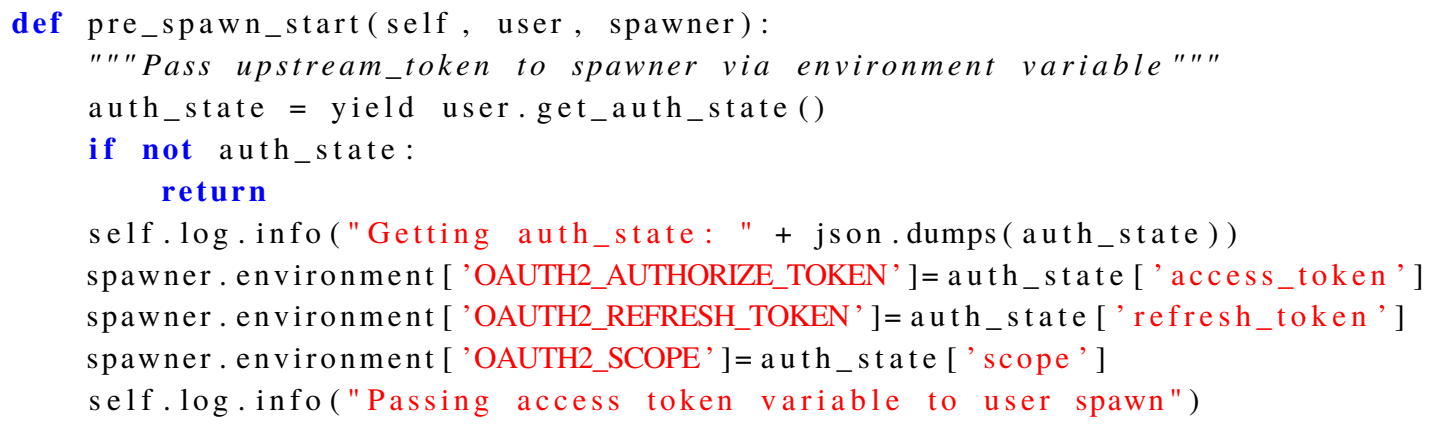

In this case, the "generic" class within the "Oautenticator" package needs to include a "pre_spawn_start" method to indicate a set of environmental variables to be sent to the docker container deployed as a user workspace (default folder: /usr/local/lib/python3.5/dist-packages/oauthenticator/generic.py). This is the key to send both the access_token and the refresh_token that will be used to access the different components as well as integrate the system. Whenever a new Docker container is launched for specific users, the tokens assigned to that user is forwarded within her/his workspace.

With all these specific configurations, the system is capable to deploy an integrated workspace for the user including the distributed storage, the access to external computing resources and the Jupyter notebooks features.

\section{Conclusions}

Applying the proposed architecture, a scalable system to satisfy the needs of different users can be provided in a Cloud Computing environment. The combined use of technologies (Docker, Open 
ID Connect) and tools (Jupyter, Onedata, PaaS Orchestrator) enables a system oriented to manage data in a virtual fashion, without the need of using local resources.

The advantages of using a distributed storage space are considerable and it offers high flexibility in terms of data management (access in different ways, mount the space only in the cloud, etc.). For those cases that the user need to process data in an exhaustive way, the PaaS Orchestrator works as a bridge between Jupyter and advanced computing sites. Everything is coherent thanks to Open ID connect tokens, which allow to keep a track of the user activities as well as keep them authenticated within the different components.

The limitation of the system is the network speed. In order to support the mounting of remote storage space, the network needs to have a minimum of bandwidth. That is why the Jupyter Hub master node and the user workspace need to be deployed in a server with High-speed internet connection (at least 10G) in order to support the data flow.

This system offers the most demanding features in Data Science and related disciplines, with access to data storage and computing in a user-friendly way thanks to Jupyter.

\section{References}

[1] Perez, Fernando and Granger, Brian E. IPython: A System for Interactive Scientific Computing, Computing in Science \& Engineering (2007).

[2] White, Jeremy T. and Fienen, Michael N. and Doherty, John E. A python framework for environmental model uncertainty analysis Elsevier (2016) DOI: 10.1016/J.ENVSOFT.2016.08.017

[3] Fernandez, Nicolas F. and Gundersen, Gregory W. and Rahman, Adeeb and Grimes, Mark L. and Rikova, Klarisa and Hornbeck, Peter and Ma'ayan, Avi Clustergrammer, a web-based heatmap visualization and analysis tool for high-dimensional biological data Nature Publishing Group (2017) DOI: $10.1038 /$ sdata.2017.151

[4] Merkel, D. Docker: Lightweight Linux Containers for Consistent Development and Deployment Linux J. 239 (2014)

[5] Sakimura, Nat and Bradley, John and Jones, Mike and de Medeiros, Breno and Mortimore, Chuck OpenID Connect Core 1.0 incorporating errata set 1 The OpenID Foundation, specification (2014)

[6] Dutka, Łukasz and Wrzeszcz, Michal and Lichoń, Tomasz and Słota, Rafał and Zemek, Konrad and Trzepla, Krzysztof and Opioła, Lukasz and Słota, Renata and Kitowski, Jacek Onedata - A Step Forward towards Globalization of Data Access for Computing Infrastructures Procedia Computer Science (2015) DOI: 10.1016/j.procs.2015.05.445

[7] Caballer, Miguel and Zala, Sahdev and García, Álvaro López and Moltó, Germán and Fernández, Pablo Orviz and Velten, Mathieu Orchestrating Complex Application Architectures in Heterogeneous Clouds Journal of Grid Computing (2018) DOI: 10.1007/s10723-017-9418-y

[8] Wilkinson, Mark D. and Dumontier, Michel and Aalbersberg, IJsbrand Jan and Appleton, Gabrielle and Axton, Myles and Baak, Arie and Blomberg, Niklas and Boiten, Jan-Willem and da Silva Santos, Luiz Bonino and Bourne, Philip E. and Bouwman, Jildau and Brookes, Anthony J. and Clark, Tim and Crosas, Mercè and Dillo, Ingrid and Dumon, Olivier and Edmunds, Scott and Evelo, Chris T. and Finkers, Richard and Gonzalez-Beltran, Alejandra and Gray, Alasdair J.G. and Groth, Paul and Goble, 
Carole and Grethe, Jeffrey S. and Heringa, Jaap and 't Hoen, Peter A.C and Hooft, Rob and Kuhn, Tobias and Kok, Ruben and Kok, Joost and Lusher, Scott J. and Martone, Maryann E. and Mons, Albert and Packer, Abel L. and Persson, Bengt and Rocca-Serra, Philippe and Roos, Marco and van Schaik, Rene and Sansone, Susanna-Assunta and Schultes, Erik and Sengstag, Thierry and Slater, Ted and Strawn, George and Swertz, Morris A. and Thompson, Mark and van der Lei, Johan and van Mulligen, Erik and Velterop, Jan and Waagmeester, Andra and Wittenburg, Peter and Wolstencroft, Katherine and Zhao, Jun and Mons, Barend The FAIR Guiding Principles for scientific data management and stewardship Nature Publishing Group (2016) DOI: 10.1038/sdata.2016.18

[9] Mons, Barend and Neylon, Cameron and Velterop, Jan and Dumontier, Michel and da Silva Santos, Luiz Olavo Bonino and Wilkinson, Mark D Cloudy, increasingly FAIR; revisiting the FAIR Data guiding principles for the European Open Science Cloud Information Services \& Use (2017)

[10] Brunner, Robert J and Kim, Edward J emphTeaching data science Procedia Computer Science (2016)

[11] Harper, D What is eutrophication? Springer Netherlands (1992) DOI: 10.1007/978-94-011-3082-01

[12] Merkel, Dirk Docker: Lightweight Linux Containers for Consistent Development and Deployment Linux J. (2014)

[13] Open Archives Initiative Protocol for Metadata Harvesting URL: https://www.openarchives.org/pmh/ (accessed on 2nd october 2019)

[14] Najafabadi, Maryam M and Villanustre, Flavio and Khoshgoftaar, Taghi M and Seliya, Naeem and Wald, Randall and Muharemagic, Edin Deep learning applications and challenges in big data analytics Journal of Big Data (2015) DOI: 10.1186/s40537-014-0007-7

[15] OASIS Topology and Orchestration Specification for Cloud Applications (TOSCA) TC | OASIS 